\title{
Structural Study of the Germanium-Aluminum-Borate Glasses by Solid State NMR and Raman Spectroscopies
}

Douglas F. Franco ${ }^{1}$, Roger Gomes Fernandes ${ }^{1}$, Silvia Helena Santagneli ${ }^{*}$, Marcos de Oliveira Jr. ${ }^{2}$, Hellmut Eckert ${ }^{2,3}$, Marcelo Nalin ${ }^{1 *}$

\footnotetext{
${ }^{1}$ Institute of Chemistry- São Paulo State University-UNESP, R. Prof. Francisco Degni, 55, AraraquaraSP, Brazil

${ }^{2}$ São Carlos Institute of Physics - São Paulo University - USP, Av. Trabalhador Sãocarlense 400, São Carlos-SP, Brazil

${ }^{3}$ Institut für Physikalische Chemie, WWU Münster, Corrensstraße 28-30, D 48149 Münster, Germany
}

\section{Supporting Information}




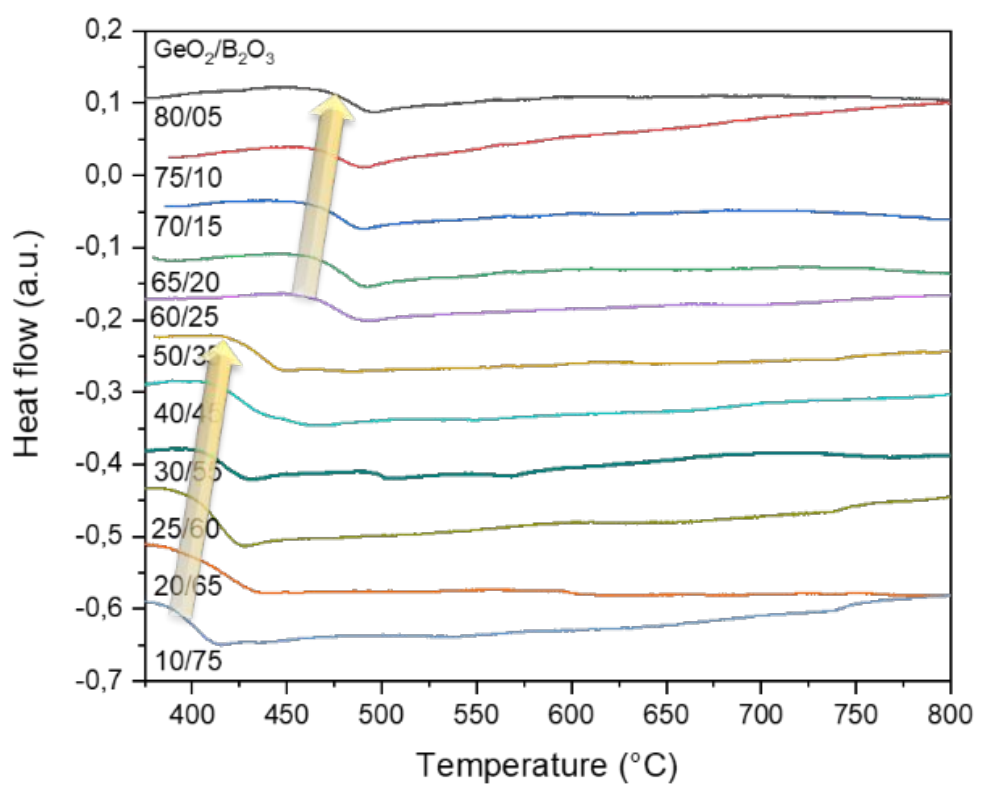

Figure $\mathrm{S} 1$. DSC cures of $(85-\mathrm{x}) \mathrm{GeO}_{2}-(\mathrm{x}) \mathrm{B}_{2} \mathrm{O}_{3}-10 \mathrm{Na}_{2} \mathrm{O}-4 \mathrm{Al}_{2} \mathrm{O}_{3}-1 \mathrm{PbO}$ glasses

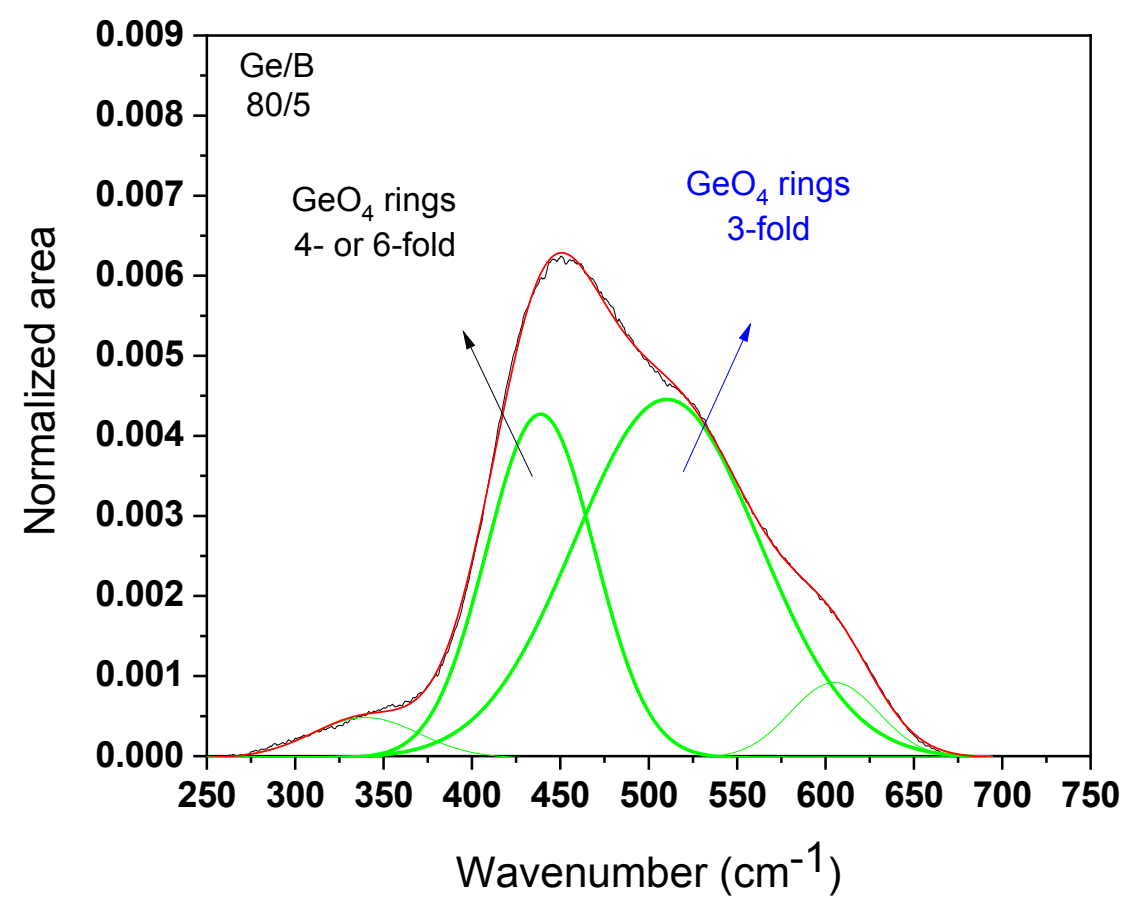



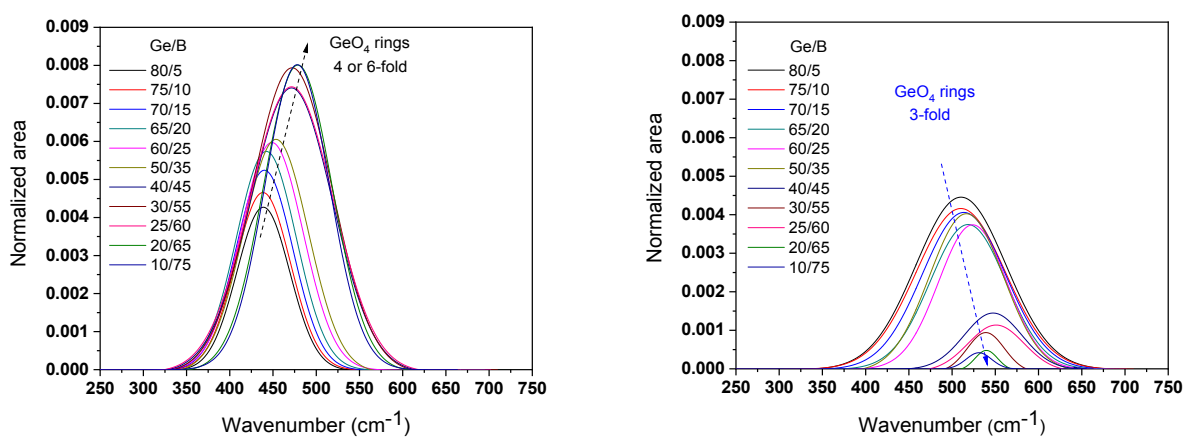

Figure S2. (online color) (Upper) Raman intensity and deconvoluted bands with area normalized for samples $80 / 05$ in the region of $250-750 \mathrm{~cm}^{-1}$. Deconvoluted bands with area normalized for all samples in the region of 350-650 $\mathrm{cm}^{-1}$ regarding $\mathrm{GeO}_{4}$ rings with 4- or 6-fold (middle) and $\mathrm{GeO}_{4}$ rings with 3-fold (bottom) bands.

Table S1: Raman deconvolution area of $(85-\mathrm{x}) \mathrm{GeO}_{2}-(\mathrm{x}) \mathrm{B}_{2} \mathrm{O}_{3}-10 \mathrm{Na}_{2} \mathrm{O}-4 \mathrm{Al}_{2} \mathrm{O}_{3}-$ $1 \mathrm{PbO}$ glasses.

\begin{tabular}{|c|c|c|}
\hline band & $\mathrm{GeO}_{4}$ rings (4- or 6- fold) & $\mathrm{GeO}_{4}$ rings (3- fold) \\
\cline { 2 - 3 } Composition & Area & Area \\
\hline $80 / 05$ & 0.317669 & 0.574723 \\
\hline $75 / 10$ & 0.364276 & 0.531466 \\
\hline $70 / 15$ & 0.425655 & 0.482604 \\
\hline $65 / 20$ & 0.491560 & 0.405305 \\
\hline $60 / 25$ & 0.534635 & 0.363032 \\
\hline $50 / 35$ & 0.538157 & 0.403399 \\
\hline $40 / 45$ & 0.852177 & 0.114923 \\
\hline $30 / 55$ & 0.891659 & 0.052654 \\
\hline $25 / 60$ & 0.875914 & 0.095420 \\
\hline $20 / 65$ & 0.779151 & 0.017368 \\
\hline $10 / 75$ & 0.748384 & 0.013819 \\
\hline
\end{tabular}



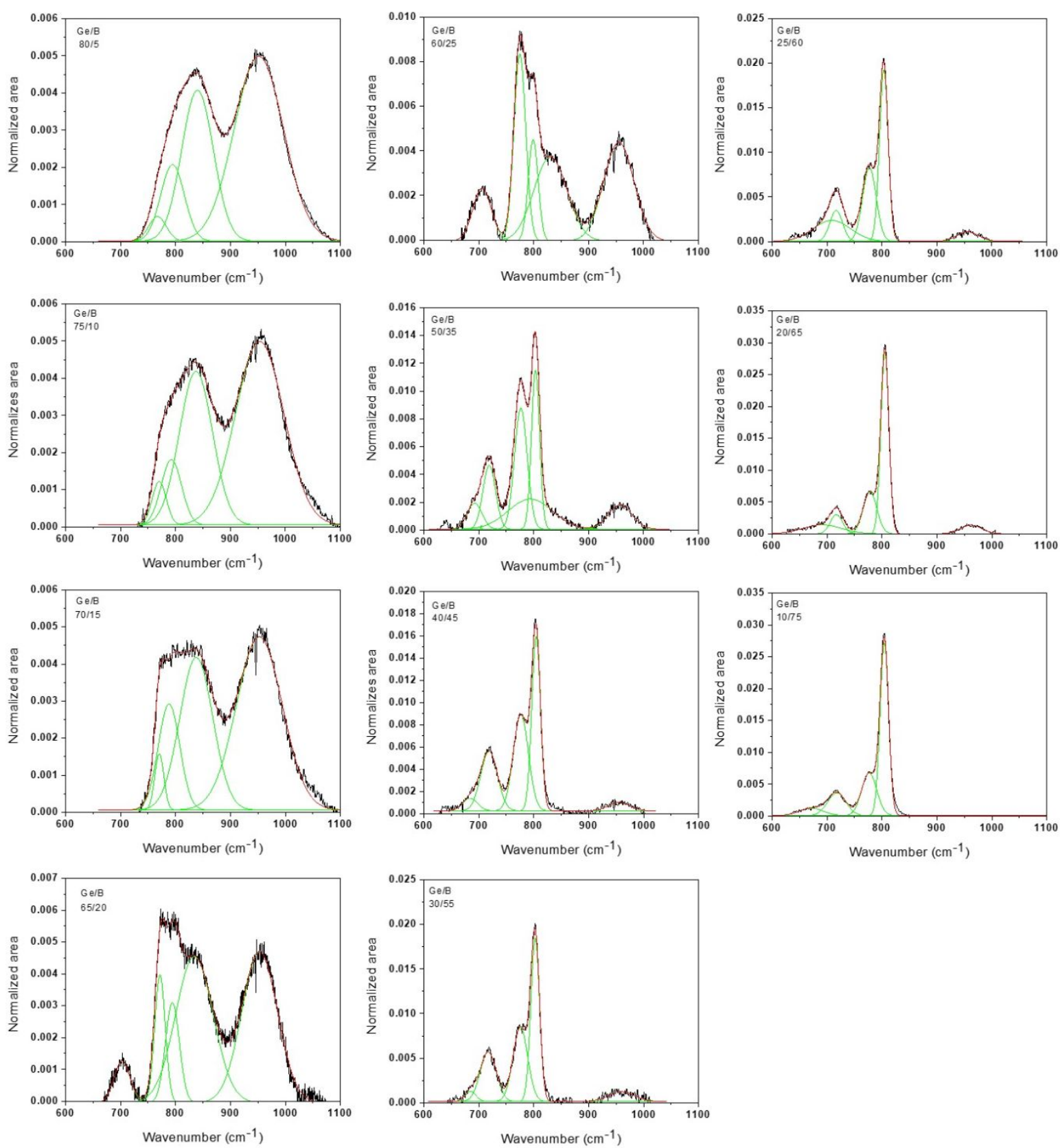

Figure S3. (online color) Raman intensity and deconvoluted bands with area normalized in the region of $650-1100 \mathrm{~cm}^{-1}$ for all glass compositions. 

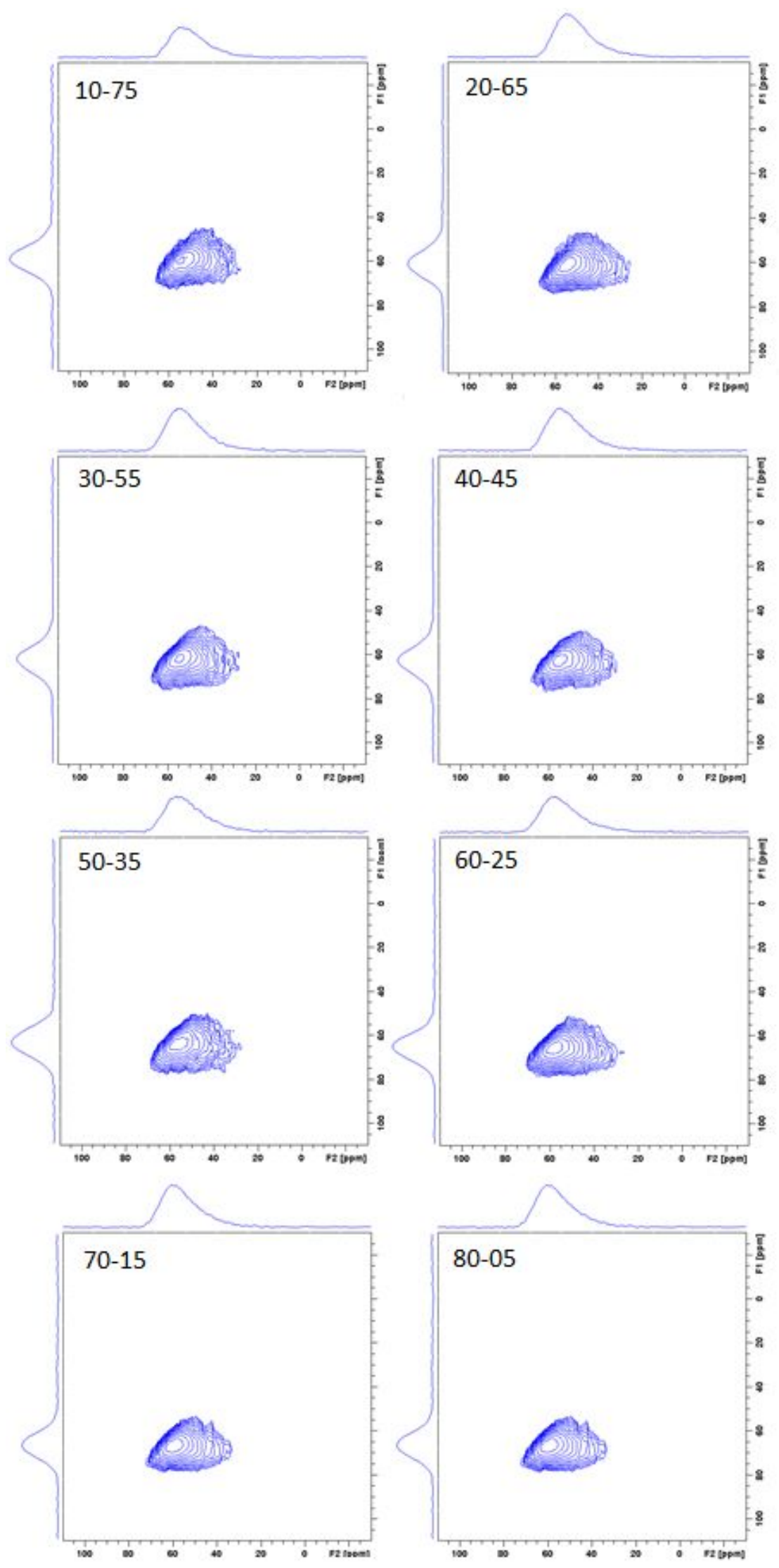

Figure S4. ${ }^{27} \mathrm{Al}$ TQMAS MAS NMR spectra of the glasses. 

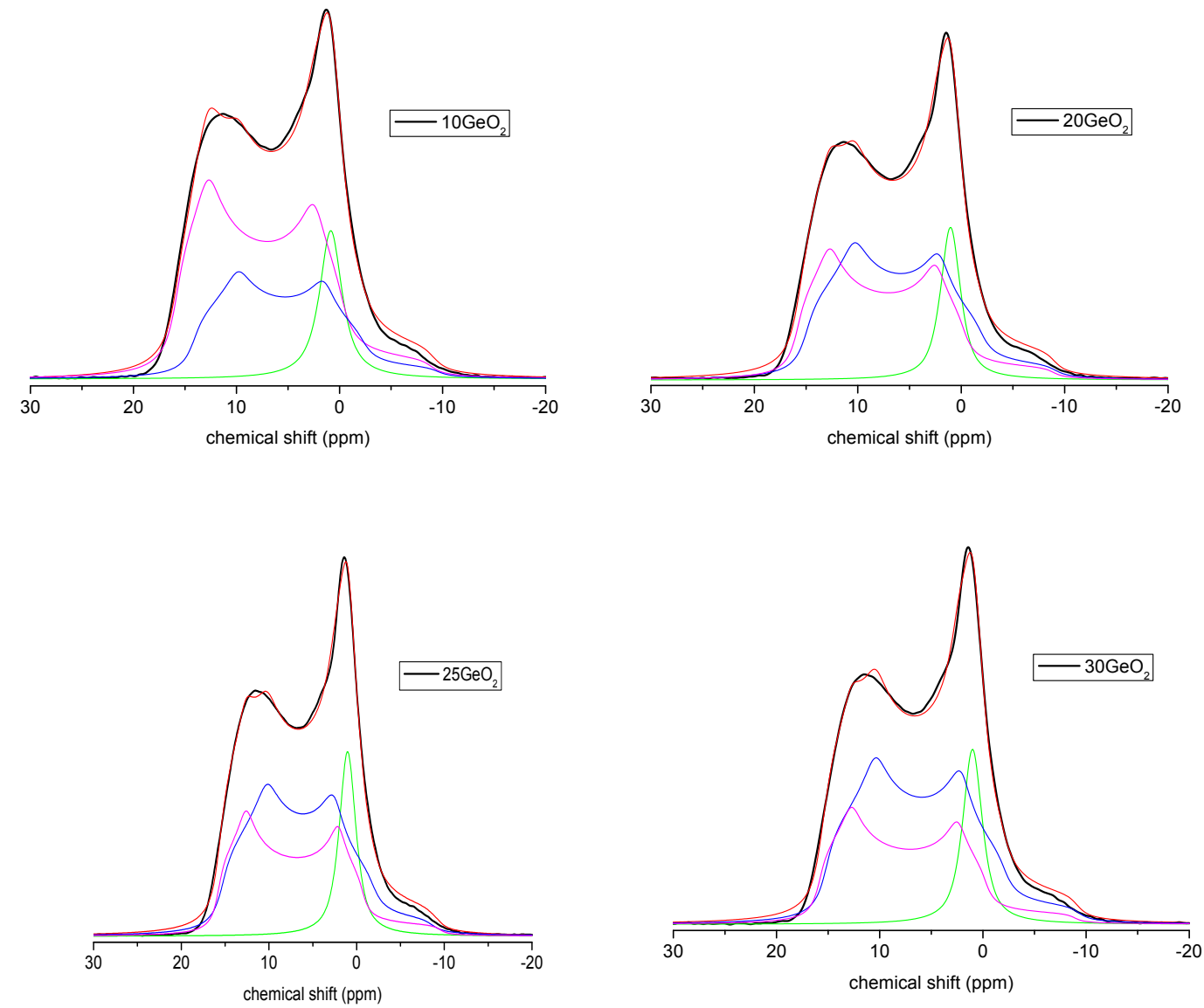

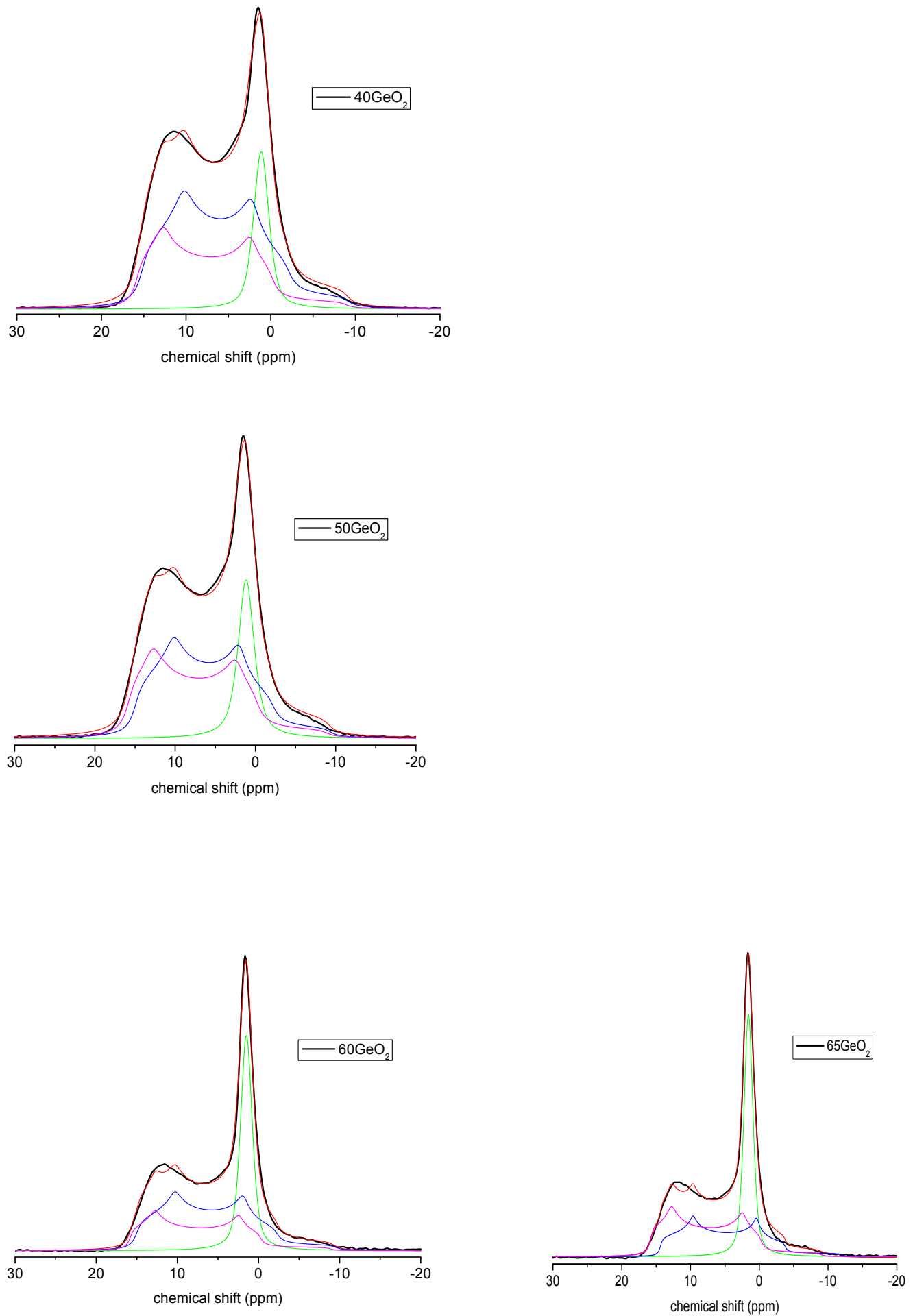

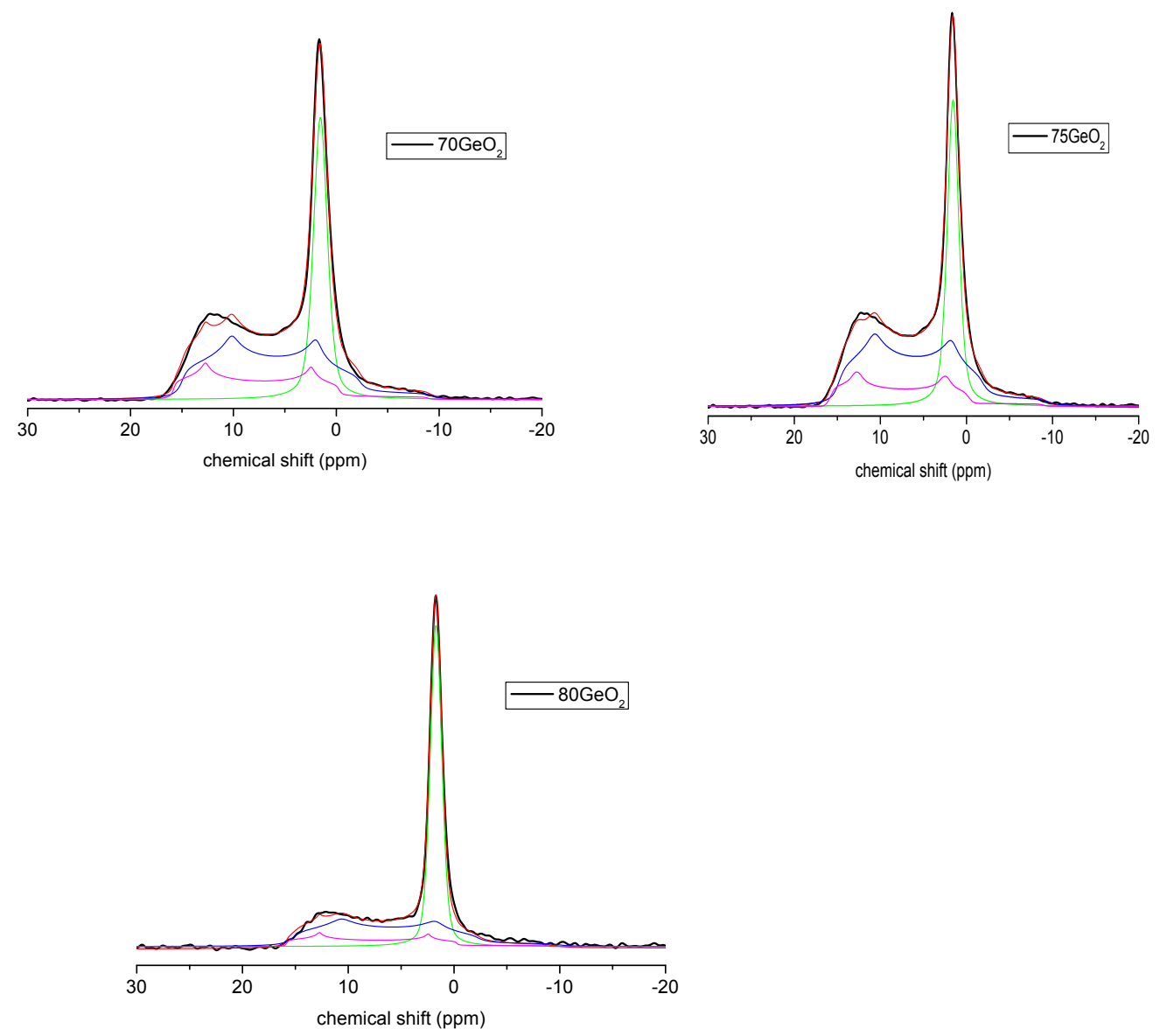

Figure S5. Deconvolution ${ }^{11} \mathrm{~B}$ MAS NMR spectra of $(85-\mathrm{x}) \mathrm{GeO}_{2}-(\mathrm{x}) \mathrm{B}_{2} \mathrm{O}_{3}-10 \mathrm{Na}_{2} \mathrm{O}-$ $4 \mathrm{Al}_{2} \mathrm{O}_{3}-1 \mathrm{PbO}$ glasses obtained with $9.4 \mathrm{~T}$.
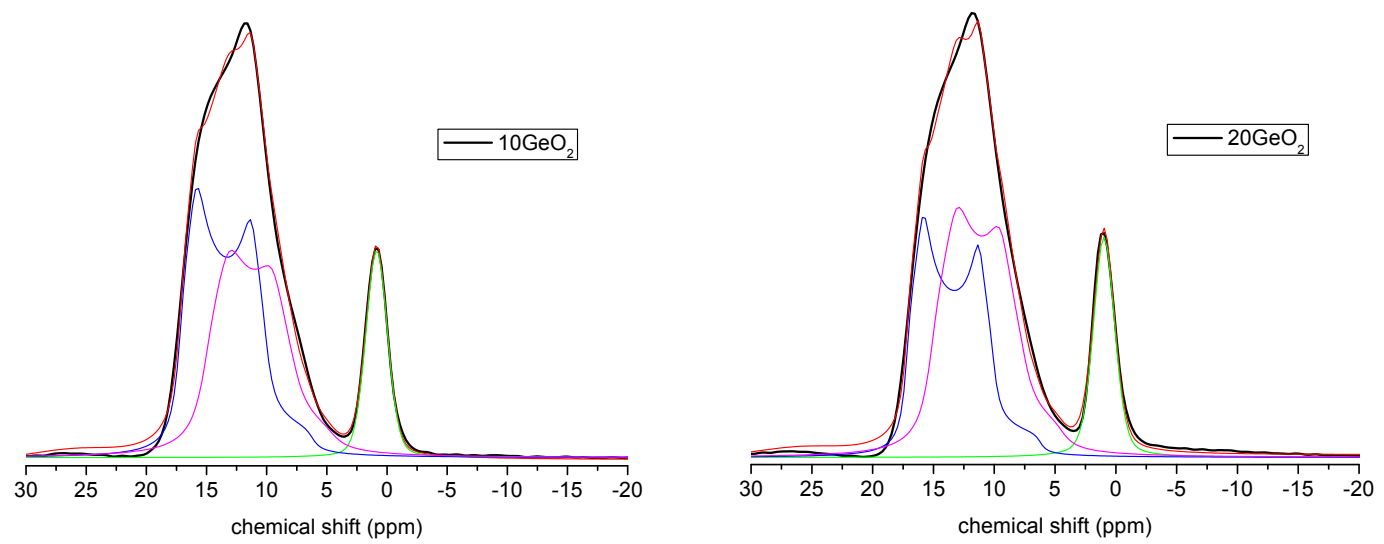

SB 

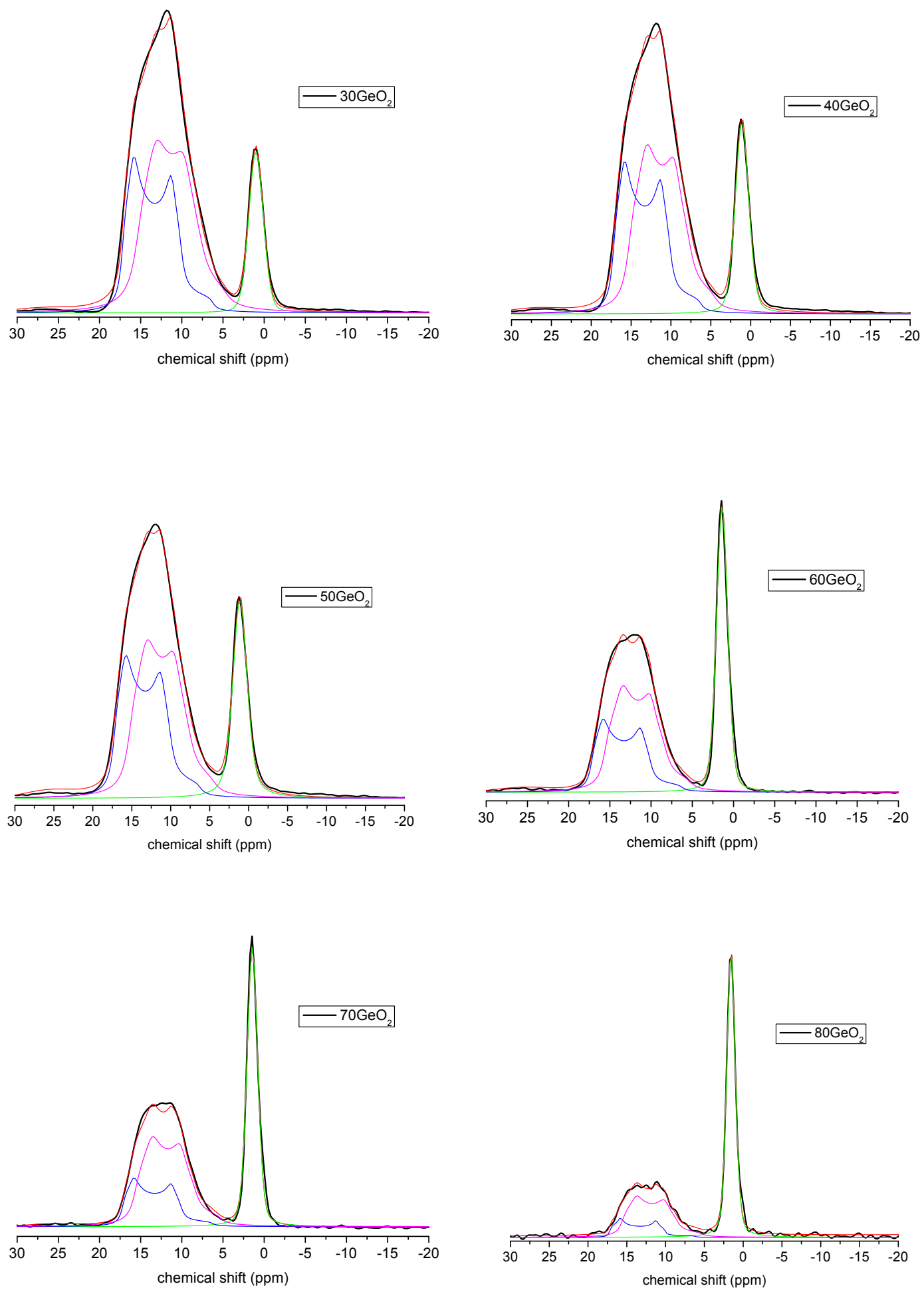

Figure S6. Deconvolution ${ }^{11} \mathrm{~B}$ MAS NMR spectra of $(85-\mathrm{x}) \mathrm{GeO}_{2}-(\mathrm{x}) \mathrm{B}_{2} \mathrm{O}_{3}-10 \mathrm{Na}_{2} \mathrm{O}-$ $4 \mathrm{Al}_{2} \mathrm{O}_{3}-1 \mathrm{PbO}$ glasses obtained with $14.09 \mathrm{~T}$. 


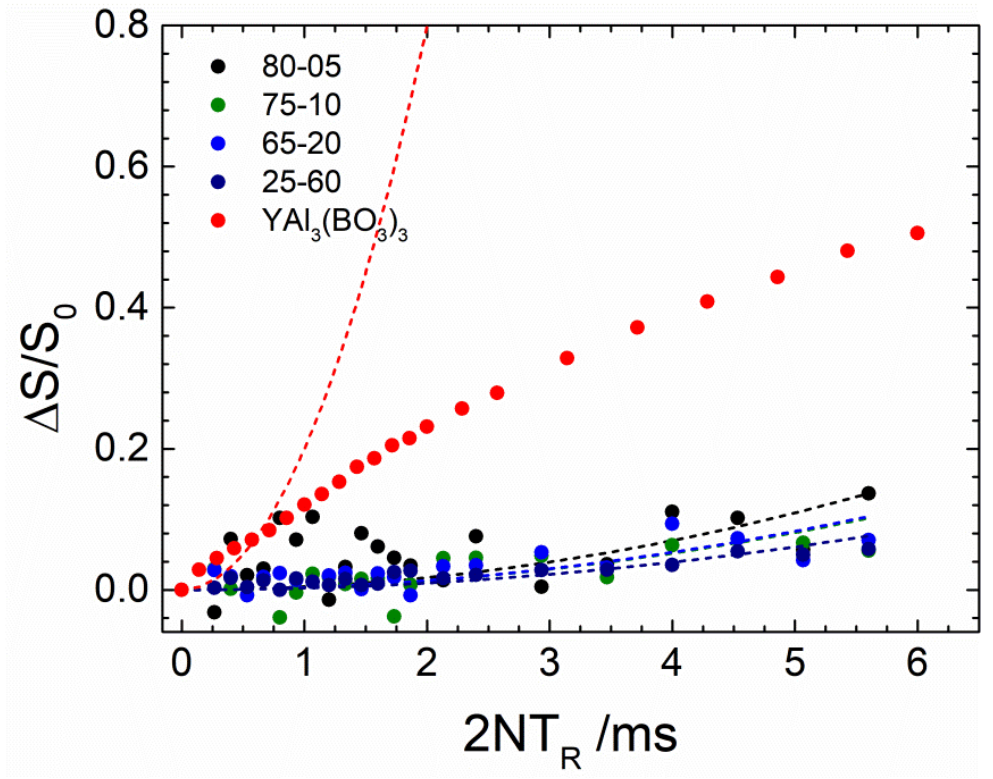

Figure $\mathrm{S} 7 .{ }^{11} \mathrm{~B}\left\{{ }^{27} \mathrm{Al}\right\}$ REDOR data measured on the glasses of the present study and the model compound $\mathrm{YAl}_{3}\left(\mathrm{BO}_{3}\right)_{4}$.
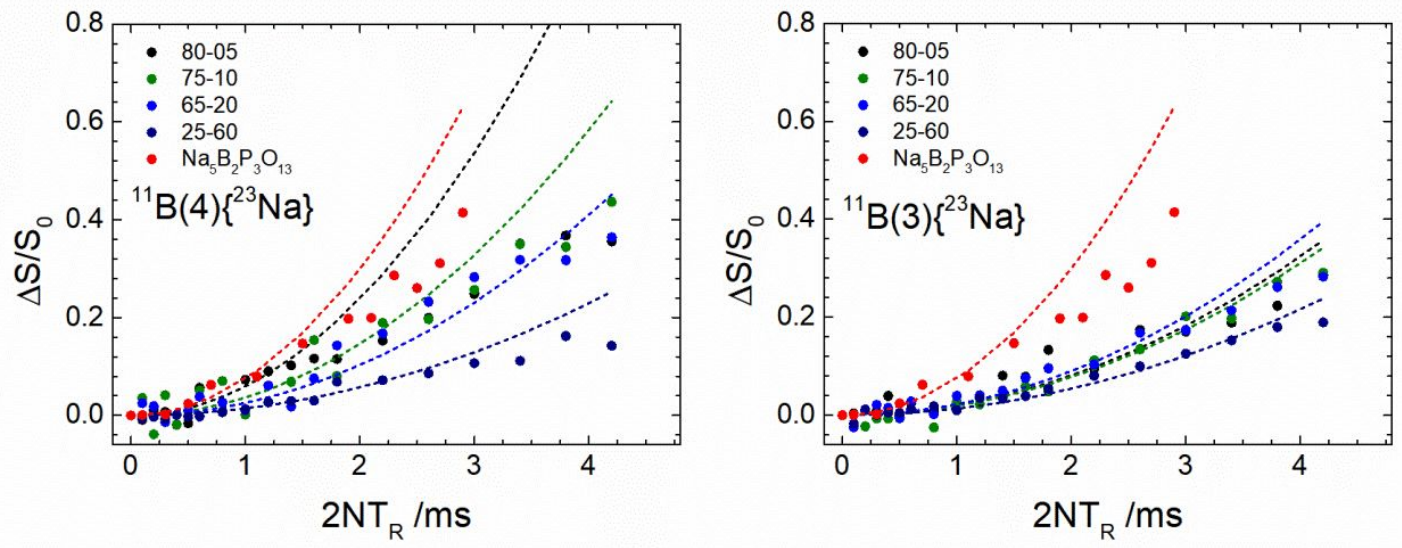

Figure S8. ${ }^{11} \mathrm{~B}\left\{{ }^{23} \mathrm{Na}\right\}$ REDOR data measured on the glasses of the present study and the model compound $\mathrm{Na}_{5} \mathrm{~B}_{2} \mathrm{P}_{3} \mathrm{O}_{13}$. Two sets of curves are shown, corresponding to experiments performed with ideal condition for three- and four-coordinated ${ }^{11} \mathrm{~B}$ species. 
Table S2. Second moments $\mathrm{M}_{2}\left(\times 10^{6} \pm 10 \% \mathrm{rad}^{2} \mathrm{~s}^{-2}\right)$ obtained from ${ }^{11} \mathrm{~B}-{ }^{23} \mathrm{Na}$ and ${ }^{11} \mathrm{~B}-{ }^{27} \mathrm{Al}$ REDOR experiments by parabolic fits of the data within the initial data range $\Delta \mathrm{S} / \mathrm{S} 0<$ 0.1 , using the equation $\Delta \mathrm{S} / \mathrm{S}_{0}=f \times \frac{1}{I(I+1) \pi^{2}}\left(N T_{R}\right)^{2} M_{2}(S\{I\})$. The correction factor $f$ was calibrated by comparison between experimental and theoretical $M_{2}$ values for crystalline model compounds.

\begin{tabular}{c|ccc|cc}
\hline Sample & \multicolumn{3}{|c|}{$\mathbf{M}_{2}\left({ }^{11} \mathbf{B}\left\{{ }^{23} \mathbf{N a}\right\}\right)$} & \multicolumn{2}{c}{$\mathbf{M}_{2}\left({ }^{11} \mathbf{B}\left\{{ }^{27} \mathbf{A}\right\}\right)$} \\
& $\mathbf{B}(3)$ & $\mathbf{B}(4)$ & $\boldsymbol{f}$ & $\mathbf{B}(3)$ & $\boldsymbol{f}$ \\
\hline 8005 & 5.7 & 17 & - & 0.2 & - \\
7510 & 5.5 & 10 & - & 0.3 & - \\
6520 & 6.4 & 7.3 & - & 0.3 & \\
2560 & 3.8 & 4.1 & - & 0.1 & - \\
$\mathrm{Na}_{5} \mathrm{~B}_{2} \mathrm{P}_{3} \mathrm{O}_{13}$ & - & $22.3^{\mathrm{a}}$ & 0.13 & - & - \\
$\mathrm{YAl}_{3}\left(\mathrm{BO}_{3}\right)_{4}$ & - & - & - & $72.3^{\mathrm{a}}$ & 0.92 \\
\hline
\end{tabular}

${ }^{a}$ Weighted average for the two B sites in the crystal structure. 